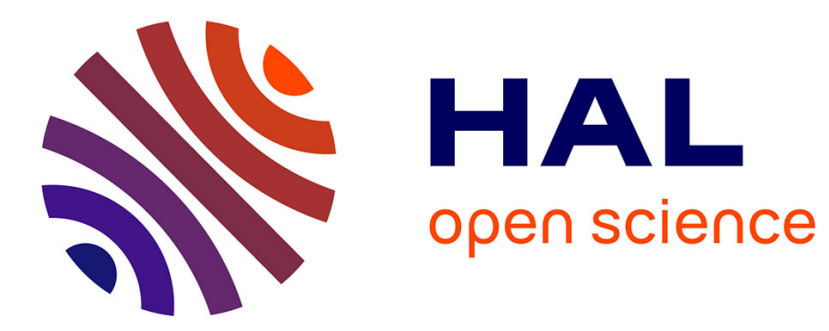

\title{
La dictature du nom. Du patronyme au pseudonyme chez les marabouts africains de Paris \\ Liliane Kuczynski
}

\section{To cite this version:}

Liliane Kuczynski. La dictature du nom. Du patronyme au pseudonyme chez les marabouts africains de Paris. L'anonymat urbain. Journée d'études de la Société d'ethnologie française (SEF) proposé par le laboratoire d'anthropologie urbaine (LAU CNRS UPR34), Petit auditorium, Musée national des arts et traditions populaires, Paris, 19 avril 1993., Apr 1993, Paris, France. halshs-00005148v2

\section{HAL Id: halshs-00005148 \\ https://shs.hal.science/halshs-00005148v2}

Submitted on 21 Aug 2006

HAL is a multi-disciplinary open access archive for the deposit and dissemination of scientific research documents, whether they are published or not. The documents may come from teaching and research institutions in France or abroad, or from public or private research centers.
L'archive ouverte pluridisciplinaire HAL, est destinée au dépôt et à la diffusion de documents scientifiques de niveau recherche, publiés ou non, émanant des établissements d'enseignement et de recherche français ou étrangers, des laboratoires publics ou privés. 


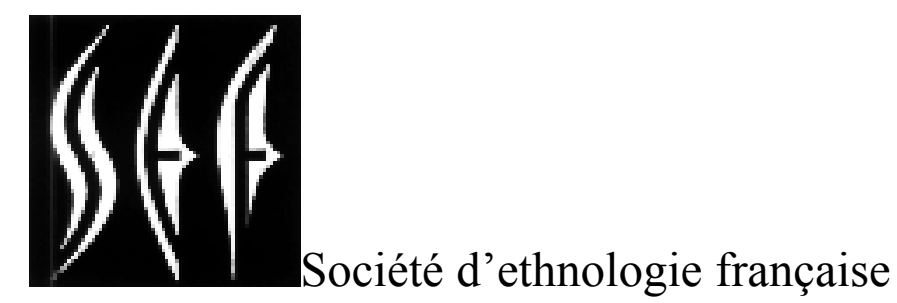

Journée d'étude du 29 avril 1993 de 10 à 17heures

proposée par le

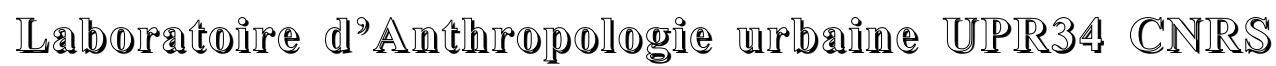

L' monymat urbain

Grand auditorium

Musée national des arts et traditions populaires 


\title{
La dictature du nom \\ Du patronyme au pseudonyme chez les marabouts africains de Paris
}

\author{
Liliane Kuczynski \\ Laboratoire d'Anthropologie urbaine
}

\begin{abstract}
S'agissant des marabouts, il semble paradoxal d'évoquer l'anonymat alors que les pratiques maraboutiques sont entièrement fondées sur la manipulation, la répétition de noms (d'Allah, d'anges, de génies...), sur la désignation (du sujet cause de l'infortune, de celui sur lequel on veut agir) et sur la reconnaissance, fûtelle fictive, du consultant par le marabout (qui dit toujours avoir su que tel devait venir le voir).
\end{abstract}

Encore faut-il qu'auparavant une rencontre ait eu lieu, une relation se soit nouée, ce qui, en milieu parisien, n'a rien d'évident. Il est incontestable qu'en France, l'effacement des signes d'identité des marabouts -l'une des facettes de l'anonymat- est plus accentuée qu'en Afrique de l'ouest.

Les sociétés sénégambiennes identifient un marabout à de multiples signes (l'âge, le regard, le vêtement, la modestie condition importante de la réputation etc.). L'appartenance lignagère, facilement connue de tous, et la place de chacun en son sein sont un facteur essentiel de reconnaissance. Le nom de lignage permet aussi de reconnaître la condition d'origine (servile, artisanale, maraboutique) de tout individu. Mais ce nom, "chose placardée" et non attribut de la personne, peut être modifié, emprunté, même si les griots ont pour charge de garder mémoire de la généalogie. Ainsi la manipulation du nom fait partie des cultures sénégambiennes.

A Paris, ces signes d'identité subissent de profondes modificactions. Pour ceux des Africains vivant en France qui accordent une certaine reconnaissance aux marabouts parisiens, certains traits ont perdu de leur pertinence tandis que d'autres (l'âge, dans une moindre mesure le nom) l'ont conservée. Mais alors qu'en Afrique, ces signes sont nécessaires et suffisants pour identifier un (bon) marabout, en France ils ne sont tout au plus que des éléments favorables qui préjugent peu d'un jugement définitif. Car la vie en France est avant tout aventure individuelle.

Ce brouillage des signes d'identité est encore plus accentué dans la perception française des marabouts et les points communs que l'on peut 
éventuellement déceler (l'importance du regard, l'âge) n'ont en rien la même valeur identificatoire. A ceci s'ajoute une perte évidente du sens du nom.

C'est précisémént cette non pertinence de certains traits, cette non fonctionnalité de l'identité lignagère, l'éparpillement parisien qui permettent, en France, à des individus d'émerger et de se forger un nom ; l'anonymat devient alors créateur.

Les moyens de se faire un nom - expression à prendre, ici, dans son sens plein - sont variés. Il y a d'abord la preuve par le témoin, qui reconnaît une efficacité personnellement éprouvée et qui inaugure une chaîne orale où le nom du marabout est répété et transmis. Il y a surtout l'usage de la publicité : elle correspond à un passage à l'écrit et à l'auto-définition, où le nom ("Monsieur Diallo") est transformé en marchandise. Cependant l'usage de cette publicité a plusieurs fonctions (notamment au regard de la légalité et de la fiscalité) qui permettent de nuancer cette affirmation.

L'analyse de la publicité, en particulier celle des noms et des titres, montre l'effort des marabouts qui y recourent pour s'insérer dans des réseaux non plus verticaux (comme le lignage) mais horizontaux (comme un appartenance professionnelle dans laquelle ils cherchent une légitimation). Quant au "bricolage du nom" proprement dit - dont on a souligné l'existence en Afrique - il révèle des manipulations à diverses fins.

S'il est vrai qu'il existe déja depuis longtemps, dans les villes sénégambiennes, des marabouts "free lance" qui cherchent à s'imposer en secouant les jougs lignagers, il est sûr que l'anonymat parisien est encore plus favorable à l'émergence de telles personnalités.

\section{Précisions 2005}

Communication publiée en 1997 : Liliane Kuczynski, «La dictature du nom. Du patronyme au pseudonyme chez les marabouts de Paris », L'Homme 141 :

101-117.

En ligne sur le site Persée :

http://www.persee.fr/showPage.do?urn=hom_0439-

4216_1997_num_37_141_370204 


\title{
Société d'ethnologie française
}

\section{Journée d'étude du 29 avril 1993 de 10 à 17heures}

\author{
proposée par le \\ Lgborgutoire dªnthropologie mrbmine CNRS

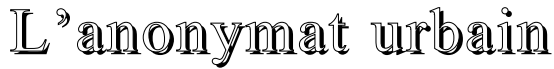

\section{Matin}

Colette Petonnet

Introduction

L'anonymat comme principe fondateur des villes http://halshs.ccsd.cnrs.fr/halshs-00004044

Didier Privat

Généalogie de la notion

http://halshs.ccsd.cnrs.fr/halshs-00089561

Daniel Terrolle Rencontres en train

http://halshs.ccsd.cnrs.fr/halshs-00089560

\section{Après-midi}

Liliane Kuczynski

La dictature du nom ; du patronyme au pseudonyme chez les marabouts africains de Paris http://halshs.ccsd.cnrs.fr/halshs-00005148

Sylvie Fainzang

Quand les alcooliques ne sont pas anonymes

Eliane Daphy Qtp://halshs.ccsd.cnrs.fr/halshs-00089562

"Merci à tous les anonymes". La vedette et les autres dans le spectacle http://halshs.ccsd.cnrs.fr/halshs-00004444

Dominique Dray

Victimes d'agresseurs anonymes : reconstitutions identificatoires http://halshs.ccsd.cnrs.fr/halshs-00089563

Yves Delaporte

Quand un entomologiste rencontre un autre entomologiste... Forme et fonction des potins dans un milieu scientifique http://halshs.ccsd.cnrs.fr/halshs-00089564

6 avenue du Mahatma Gandhi - 75116 Paris - Petit auditorium 